\title{
Depth of Maximum Development of Extensive Air Showers by Radio Emission Data at Yakutsk EAS Array
}

\author{
Stanislav Knurenko \\ Yu. G. Shafer Institute of Cosmophysical Research and Aeronomy. \\ E-mail: knurenkodikfia.sbras.ru

\section{Igor Petrov*} \\ Yu. G. Shafer Institute of Cosmophysical Research and Aeronomy. \\ E-mail: igor.petroveikfia.sbras.ru
}

\section{Zim Petrov}

Yu. G. Shafer Institute of Cosmophysical Research and Aeronomy.

E-mail: pze@ikfia.sbras.ru

\begin{abstract}
Radio emission at Yakutsk Array registers at frequency $32 \mathrm{MHz}$ and radio antennas co-located with scintillation and Cherenkov detectors of Yakutsk Array. The co-location with particle detectors brings as a profit the reconstruction of fundamental air shower parameters, such as shower axis, energy and arrival direction (azimuthal and zenith angles). The paper presents data obtained in the new series of measurements of Yakutsk Radio Array in the period 2009-2013 years. We analyze individual showers and reconstruct $\mathrm{X}_{\max }$ by slope of radio LDF. Obtained results of $\mathrm{X}_{\max }$ are compared with results of other arrays.
\end{abstract}

The 34th International Cosmic Ray Conference,

30 July- 6 August, 2015

The Hague, The Netherlands

${ }^{*}$ Speaker. 


\section{Introduction}

In the paper $[1,2,3]$ pointed out the possibility of registration of the air showers (EAS) with energy more than $10^{19}$ by radio emission arrays located at the Earth surface or by registering radio emission of air showers from satellites on the Earth orbit. MSU (Moscow State University) arrays earlier at low energies and Yakutsk array later at high energies proved that air shower radio emission can be registered by surface detector arrays which registers electron and muon component of EAS $[4,5]$.

Subsequent measurements of radio emission at the Yakutsk arrays has been shown that the radio emission from the relativistic part of the shower at energies above $10^{17} \mathrm{eV}$ can be used to study the physics of the EAS as an independent observation method and in conjunction with other methods of registration of EAS for mutual calibration [6 - 10].

In this paper we continue the analysis of the data obtained in the new series of measurements for the period 2009 - 2013 years.

\section{Measurement results}

\subsection{Lateral distribution of radio emission}

Radio showers are selected by trigger of scintillation and Cherenkov detectors of Yakutsk array. Air shower axis reconstructed separately by the data of the scintillation or Cherenkov detectors by maximum likelihood method. Energy of showers estimated by the radio pulse amplitude, according to [10].

First of all we analyzed individual LDF and derived approximation formula:

$$
\varepsilon(E, \theta, R)=(15 \pm 1)(1-\cos \theta)^{1.16 \pm 0.05} \exp \left(-\frac{R}{350 \pm 25.41}\right) \cdot\left(E_{0} / 10^{17} \mathrm{eV}\right)^{0.99 \pm 0.04}
$$

This formula well describes attenuation of radio signal amplitude with the distance from air shower axis. For example, in Fig. 1 dots represent amplitudes registered by antennas at different distances from air shower axis, with zenith angle $\theta=46.6^{\circ}$ and energy equal $\sim 10^{19} \mathrm{eV}$. Approximation (2.1) is shown in Fig. 1. by curved line. It draws attention to the presence of a signal at a great distance from the shower axis. But in this case, the signal $\varepsilon(E, \theta, R)$ was very close to noise level therefore it should be excluded from the analysis or we should have taken into account the noise influence like in [10]. For this reason, we selected for analysis air showers with signal amplitude exceeded the noise signal 5 times or more, i.e. showers with axis within the distances of $50-500 \mathrm{~m}$, depending on the shower energy. For the showers with energy more than $3 \cdot 10^{1} 8 \mathrm{eV}$ distance interval is shifted to higher $\mathrm{R}$.

Function of LDF for a large amount of showers normalized to a single energy and with zenith angles $\theta \leq 40^{\circ}$, is shown in Fig. 2. Also, for comparison LOPES data are presented [12]. If we convert data to the same energy, then there is a good agreement in shape of LDF and signal amplitude at given distance. Practically, this means that the calibration of measurements and energy of EAS estimation of both arrays are close. It means that other characteristics of air showers, obtained from radio emission measurement will be close as well. 


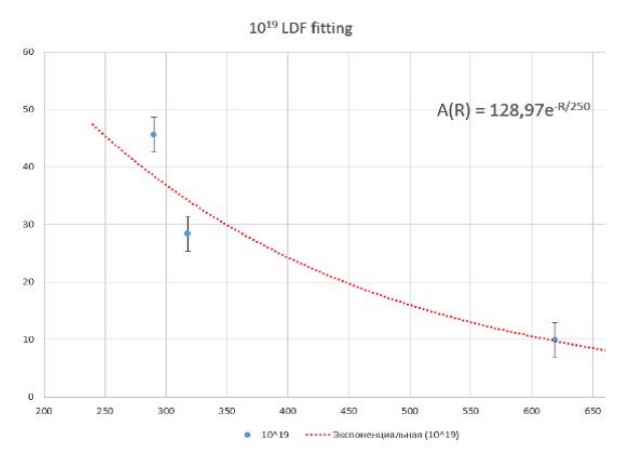

Figure 1: Dependence of radio emission amplitude $\varepsilon(\mathrm{R}, \theta)$ of individual air shower with the distance to the axis of EAS.

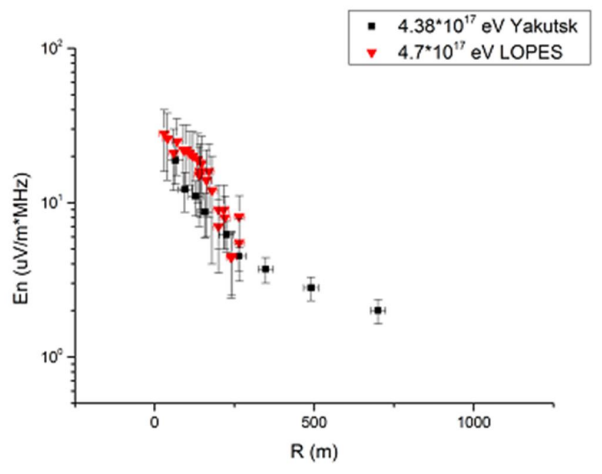

Figure 2: Average LDF (black dots) by Yakutsk and comparison with LOPES data (red dots).

\section{$2.2 X_{\max }$ by air shower radio emission data}

Taking model calculation [10] of connection between LDF and empirically derived correlation $X_{\max }$ with LDF as a basis, we estimated $X_{\max }$ in showers with energies $10^{17}-10^{19} \mathrm{eV}$ and then grouped them by energies in a few ranges. Averaging was done with taking into account obtained dependence of $X_{\max }$ from energy. The results are shown in Fig. 3, together with the result obtained at the Moscow State University [13] and LOPES [12]. There are calculations for the model QGSjet II-04 made for proton nuclei of carbon and iron nuclei. According to Fig. 4, not all data obtained with good accuracy, because of small statistics. Despite this, the data indicate a rapid advance of $X_{\max }$ above $2 \cdot 10^{17} \mathrm{eV}$. Comparison of the data with calculations said that the mass composition of primary particles in this energy range must be dominated by light nuclei.

$$
<\ln A>=\left[\left(X_{\max }^{\exp }-X_{\max }^{p}\right) /\left(X_{\max }^{F e}-X_{\max }^{p}\right)\right] \cdot \ln 56
$$

Fig. 3 shows the data for mass composition of cosmic rays obtained experimental data interpolation Yakutsk (Fig. 4) according to the following formula (2.2) and models QGSJET II-04, designed for primary protons and iron nuclei. Fig. 5 also shows the data obtained by measuring the radio emission at LOPES and MSU arrays. They are in sufficient agreement within $X_{\max }$ estimation methodology and statistical errors. For comparison Fig. 4 also shows $X_{\max }$ data obtained from Cherenkov light measurements [14]. From Fig. 5 it is clear that the new data on the mass composition is not in a contradiction with our conclusion $[15,16]$ of a sudden change in the mass composition in the energy $8 \cdot 10^{16}-3 \cdot 10^{17} \mathrm{eV}$, i.e. increase the proportion of light nuclei in cosmic rays above $\sim 3 \cdot 10^{17} \mathrm{eV}$.

\section{Conclusion}

Radio emission measurement of ultra-high energy air showers at Yakutsk in 2009-2013 shown: Air showers with energy above $10^{19} \mathrm{eV}$ can be registered by radio method and can be used as independent method to study ultra-high energy showers. 


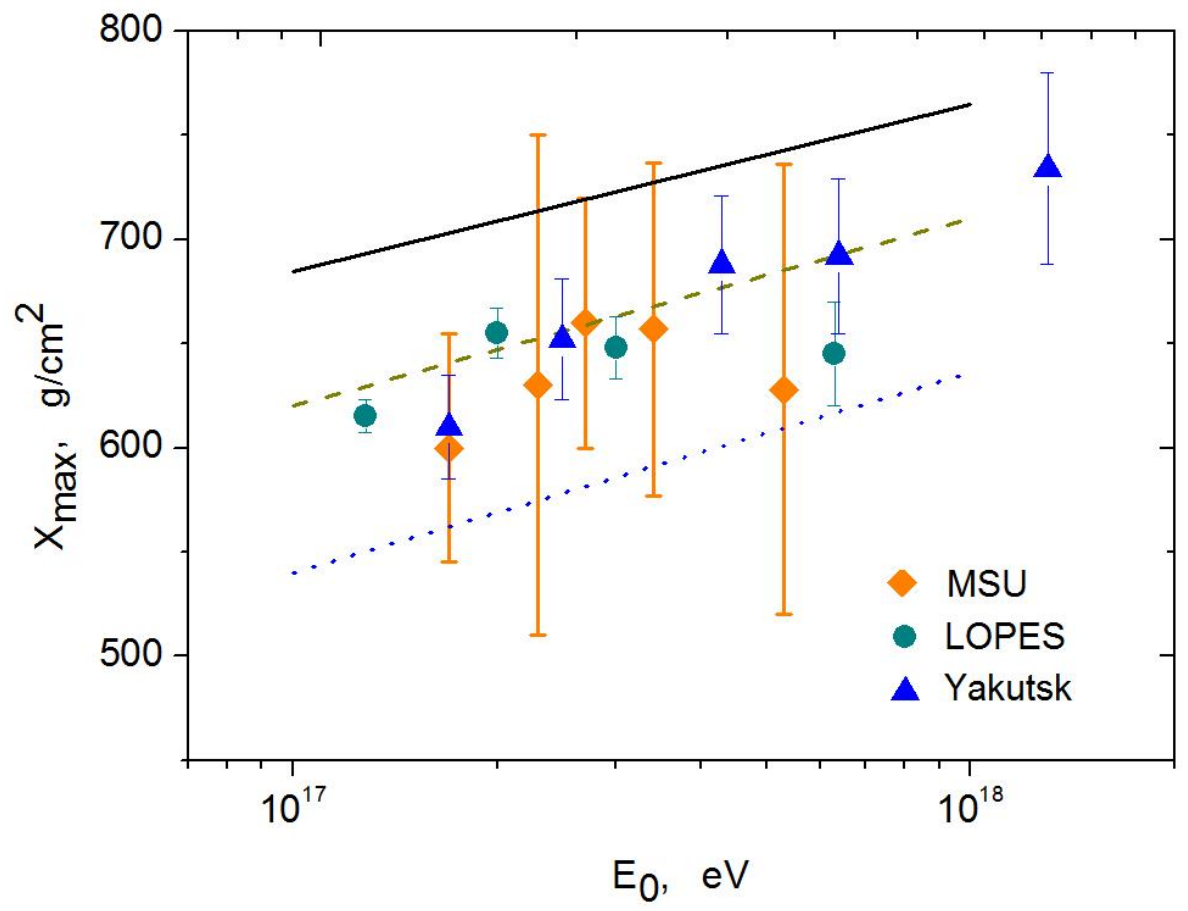

Figure 3: Comparison of data $X_{\max }^{\text {rad }}$ of Yakutsk (triangles), MSU (diamonds) and LOPES (dots).

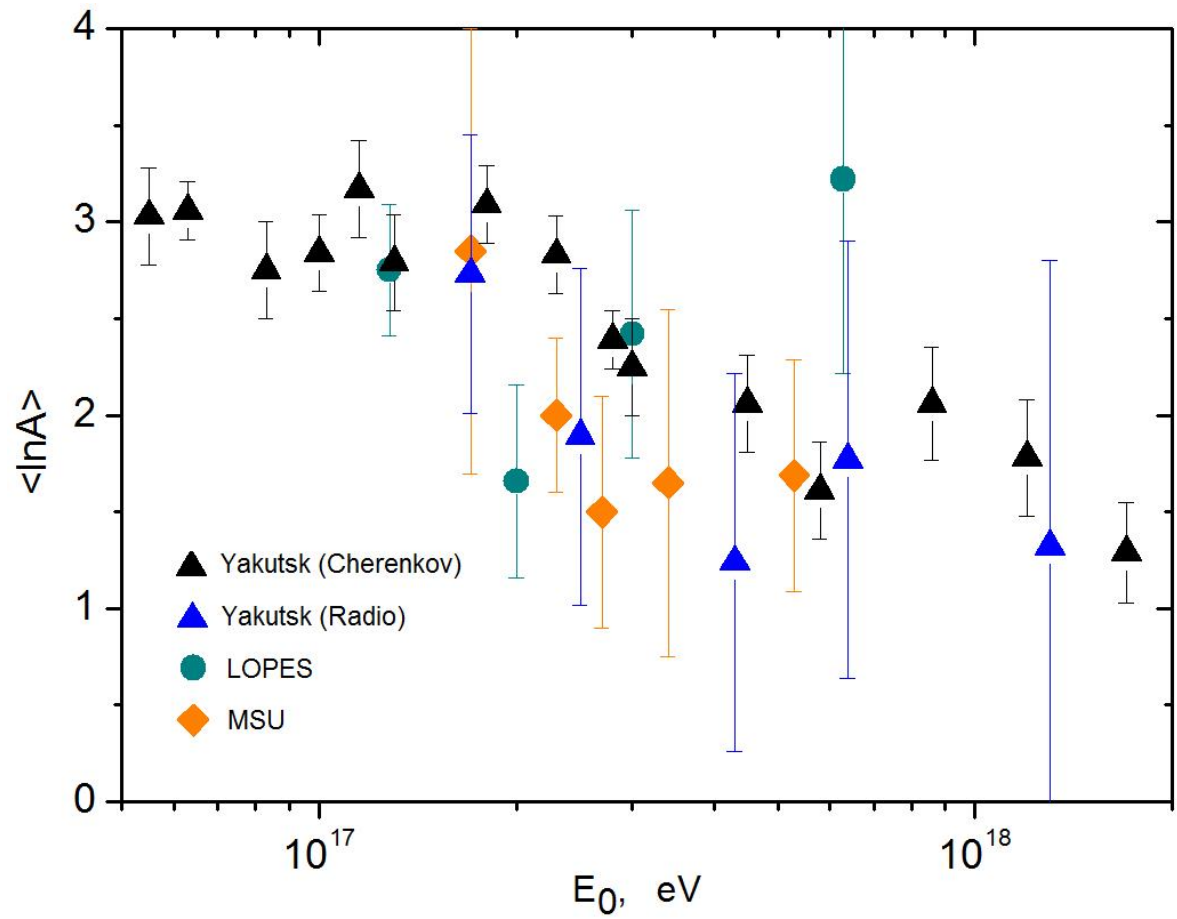

Figure 4: Dependence of $<\ln A>$ from air shower energy by radio emission and Cherenkov light data of Yakutsk array (black triangles - Cherenkov method, blue - radio methods), LOPES array (dots) and MSU (diamonds). 
The results of $X_{\max }$ obtained from radio measurements in energy range $10^{17}-10^{18} \mathrm{eV}$, despite big statistical uncertainty, yet indicate a trend of rapid advancement of $X_{\max }$ above $3 \cdot 10^{17} \mathrm{eV}$.

In the frame of QGSJET II-04 model and using method (2.2) by Yakutsk data, we obtained estimation of cosmic ray mass composition in the energy range $10^{17}-10^{18} \mathrm{eV}$, which along with the data of other arrays does not contradict our results [16], obtained from Cherenkov light measurements. In Fig. 4 all data indicate change of mass composition of cosmic rays with energy above $3 \cdot 10^{17} \mathrm{eV}$, from heavy to light nuclei.

\section{References}

[1] S. N. Vernov, A. T. Abrosimov, V. D. Volovik et al. JETP Letter, 157-162 (1966)

[2] A. D. Filonenko. UFN, V. 172, 4, 439-471 (2002)

[3] V. A. Tsarev. Elementary particle \& atomic nucleus Physics, V. 35, 1, 1-49 (2004)

[4] V. B. Atrashkevich, O. V. Vedeneev, Ch. R. Allan et al. Nuclear Physics, V. 28, 3, 712 (1978)

[5] V. P. Artamonov, V. B. Atrashkevich, T. A. Egorov et al. SUCCR (Alma-ata), 47-48 (1988)

[6] V. P. Artamonov, T. E. Egorov, A. N. Efimov et al. Proc. 21 ICRC (Adelaide), V. 9, 210 (1990)

[7] Z. E. Petrov, I. S. Petrov, S. P. Knurenko et al. NEFU Bulletin, V. 8, 4, 5-10 (2011)

[8] S. P. Knurenko, V. I. Kozlov, Z. E. Petrov and M. I. Pravdin. J. Phys.: Conf. Ser., 409, 012070 (2013). doi:10.1088/1742-6596/409/1/012070

[9] S. P. Knurenko, D. S. Borschevsky, Z. E. Petrov and I. S. Petrov. Proc. SPIE, 8696, 86960Q (2012). doi: $10.1117 / 12.2008640$.

[10] S. P. Knurenko, I. S. Petrov. RAS Bulletin. Phys. Ser. V. 79, 3, 448-450 (2015)

[11] Horneffer et al. Proc. 30 ICRC (Merida), V. 4, 83 (2007)

[12] W. D. Apel et al. arXiv: 1408.2346v1 [astro-ph. IM] 11 Aug 2014

[13] N. N. Kalmykov, A. A. Konstantinov, O. V. Vedeneev. J. Phys.: Conf. Ser.V, 409 (2012)

[14] E.G. Berezhko, S.P. Knurenko, L.T. Ksenofontov. Astrop. Phys., 36, 31-36 (2012)

[15] S. P. Knurenko et al. JETP Letters, V. 83, 11, 563 (2006)

[16] S.P. Knurenko, I.S. Petrov. Proc. ISVHECRI 2014 (in press) 